\title{
Erratum: miR-24 limits aortic vascular inflammation and murine abdominal aneurysm development
}

Lars Maegdefessel, Joshua M. Spin, Uwe Raaz, Suzanne M. Eken, Ryuji Toh, Junya Azuma, Matti Adam, Futoshi Nakagami, Helen M. Heymann, Ekaterina Chernogubova, Hong Jin, Joy Roy, Rebecka Hultgren, Kenneth Caidahl, Sonja Schrepfer, Anders Hamsten, Per Eriksson, Michael V. McConnell, Ronald L. Dalman \& Philip S. Tsao

Nature Communications 5:5214 doi: 10.1038/ncomms6214 (2014); Published 31 Oct 2014; Updated 26 Feb 2015

The original version of this Article contained a typographical error in the spelling of the authors Futoshi Nakagami and Ekaterina Chernogubova, which were incorrectly given as Futoshi Nagakami and Ekaterina Chernugobova. This has now been corrected in both the PDF and the HTML versions of the Article. 